\title{
Surveillance of antimalarial drug resistance in China in the 1980s-1990s
}

De-quan Liu

\begin{abstract}
Since the successful preparation of the microplates and the medium for field application, the resistance degree and its geographical distribution of chloroquine-resistant Plasmodium falciparum, the fluctuation of the resistance degree of $P$. falciparum to chloroquine, and the sensitivity of the parasite to commonly used antimalarial drugs were investigated between 1980 and 2003 by the in vitro microtest and the in vivo four-week test recommended by the World Health Organization (WHO). The results indicated that chloroquine-resistant falciparum malaria was present in all eight provinces/autonomous regions endemic for falciparum malaria in China, and the resistance was high and widely distributed in the Hainan and Yunnan provinces. When the use of chloroquine was stopped or administered in a decreased quanity, the drug resistance gradually decreased. In Hainan and Yunnan, P. falciparum was still highly resistant to chloroquine, amodiaquine and piperaquine, and sensitive to pyronaridine and artemisinin derivatives, but the sensitivity was gradually reduced. Based on these results, principles and therapeutic regimens for antimalarial drug use in China were formulated, the use of the antimalarials which had already developed resistance was stopped or reduced, and recommendations to use artemisinin derivatives or compound pyronaridine to promote a rational use of antimalarials and strengthen malaria control were made. The results showed that malaria incidence had declined, and endemic areas of falciparum malaria have been gradually reducing since the mid-1980s.
\end{abstract}

Keywords: Plasmodium falciparum, Resistance, Sensitivity, Antimalarial drugs, in vivo, in vitro, Surveillance

\section{Multilingual abstracts}

Please see Additional file 1 for translations of the abstract into the six official working languages of the United Nations.

\section{Review}

Both P. falciparum and P. vivax can develop drug resistance, but the resistance is much more important in $P$. falciparum, not only for its high rate and degree of resistance and wide geographical distribution, but also for its ability to cause high mortality. The resistance of $P$. falciparum to quinine, proguanil and cycloguanil, pyrimethamine, chloroquine was reported in the early $20^{\text {th }}$ century, late $1940 \mathrm{~s}$, mid-1950s and late 1950 s respectively. By the end of the 1950s, chloroquine-resistant malaria cases were found in Colombia and Thailand. Since then, the chloroquine resistance of the parasite rapidly spread to almost all falciparum malaria endemic areas

Correspondence: dqliu2002@163.com

National Institute of Parasitic Diseases, Chinese Center for Disease Control and Prevention, 200025 Shanghai, China except for Central America [1], and multiple drug resistance appeared [2,3]. Presently, P. falciparum has developed resistance to almost all available antimalarial drugs. In recent years, $P$. vivax has also developed resistance to chloroquine [4,5]. The development of drug resistance of malaria parasites is faster than any new drug development. It has become a worldwide problem, causing more serious malaria transmission in some areas or countries, recurrence of malaria transmission in areas where malaria transmission was once interrupted, and a significant increase of morbidity and mortality, as well as noticeable increases in the cost of malaria control. Resistance of $P$. falciparum to pyrimethamine, proguanil, and cycloguanil has long been reported, but resistance to chloroquine was reported only at later stages. Chloroquine resistance of $P$. falciparum was found in the Yunnan Province [6,7] and the Hainan Province in 1973 [8,9] and 1974, respectively. Since then, it has spread widely throughout the endemic areas of falciparum malaria in China in the late 1970 s to early 1980 s, and either resistance to new antimalarials appeared or sensitivity to drugs 
reduced gradually, resulting in an increase of malaria cases and posing a major challenge for malaria control. Therefore, drug resistance of falciparum malaria became an urgent issue to tackle in malaria control in China. Before 1978, the WHO standard in vivo tests [10] (seven-day or four-week tests) were used for the assessment of drug resistance, but the tests are time consuming and costly, the patients need to be hospitalized (and are usually not compliant), and the result of assessment is affected by the patients' immunity, thus it is not applicable in large areas. In 1979, an in vitro microtechnique was recommended by the WHO to assess drug resistance of P. falciparum, which was simpler, fast, accurate and acceptable, and therefore appropriate to use in a large-scale survey $[11,12]$. The WHO had published updated protocol for antimalarial drug resistance surveillance in vivo (2003), in vitro (2001), and genotyping method (2007) [13-15]. In 1978, the authors had successfully established in vitro continuous culture of erythrocytic stage $P$. falciparum, which provided an appropriate environment for the development of the in vitro microtechnique, including the drug-coated plates and the medium. Based on these technical progresses [16,17], a large-scale assessment of drug resistance was carried out by using both in vivo and in vitro tests between 1980 and 2003. The results of the investigation were highly beneficial to advise on using antimalarial drugs rationally in the malaria control program.

\section{Developing an in vitro microtechnique for determining the sensitivity of $P$. falciparum to antimalarial drugs}

To undertake a field survey by the in vitro microtechnique, it is necessary to first prepare a drug-coated plate and a medium, both of which are applicable in the field. The WHO developed a standard chloroquine-coated plate and test kit in 1979 [12]. After a trial application, we found that its medium must be made into a culture solution in the field using a relatively cumbersome procedure. In addition, it is easy to pollute, its success rate is low, and the effective period is only 48 hours at $4^{\circ} \mathrm{C}$. The maximum dosage of chloroquine in a chloroquine-coated plate was $32 \mathrm{pmol} /$ well, which could not inhibit the growth and development of $P$. falciparum completely, and it had only one control well for observing the growth and development of the parasite. The WHO chloroquine-coated microplate had 96 wells for testing 12 cases; therefore each plate must be put into an incubator repeatedly, which may cause an error between the before and after results. In order to promote the application of the technique to better understand the situation on the drug sensitivity of P. falciparum in China, we started to develop drug-coated plates and field applicable mediums in 1979.
A homemade plastic plate suitable for malaria parasite growth was selected to make the chloroquine-coated microplate. Each plate had four horizontal rows with 10 wells in each row, a total of 40 wells. The size and depth of the well were the same as in the WHO plate. The chloroquine diphosphate solution was filled into the wells with reference to the dosage of the WHO chloroquinecoated microplate, two more wells were added-one for higher dosage and one for blank control [16] —which were kept in an incubator for 24 hours, the wells were sealed with plastic adhesive tape after drying, the drug-coated plate was put into a plastic bag, and stored at room temperature. Both laboratory and field tests indicated that the plate was as effective as the WHO standard plate, suitable for field use in areas with higher chloroquine resistance, and could provide a timely observation on the growth and development of malaria parasites. All these factors resulted in the test success rate increasing. When stored at room temperature for over a year, the effect remains the same $[18,19]$.

Based on the successful preparation of the chloroquinecoated plate, other antimalarial drug-coated plates were also developed, including piperaquine, pyronaridine, artesunate, dihydroartemisinin, artemether, and arteether. Consequently, we could make simultaneous determinations of the sensitivity of one patient's blood to different antimalarials, and overcome the adverse effects on the growth of malaria parasites due to repeatedly entering the incubator and sealing with plastic tape. At present, the in vitro microtechnique can be used to determine the sensitivity of the malaria parasite to all commonly used antimalarials.

Based on the experience with the in vitro continuous culture of $P$. falciparum, and by carrying out repeated tests and improvements, the liquid medium was prepared with RPMI1640 powder, adding 15\% type AB human serum, then packed into sterile ampoules with $0.9 \mathrm{ml}$ of the solution in each; then after lyophilization, the ampoule was sealed and stored at $4^{\circ} \mathrm{C}$. Before use, it was dissolved with $0.21 \%$ sodium bicarbonate solution (in ampoule). This medium was plainly packed, easily prepared, and used in field situations; one ampoule of medium for one sample. Comparative tests demonstrated that the above homemade lyophilized medium was better than the WHO standard medium in supporting the growth of malaria parasites, and the effect was kept stable for two years at $4^{\circ} \mathrm{C}[16,18,19]$.

Since the preparation of lyophilized medium was still troublesome, ampoule-sealed liquid medium was prepared later. Once it was opened, it could be immediately used for testing, was much easier to apply in the field, and could remain in its effective state for two months at $4^{\circ} \mathrm{C}$. It was well accepted by various institutions in the country together with the drug-coated plates [17]. 
The chloroquine-resistance degree of $P$. falciparum and its geographical distribution

A large-scale survey on the chloroquine resistance of P. falciparum was then carried out in the 1980s in eight provinces/autonomous regions where falciparum malaria was prevalent, namely Yunnan, Hainan, Guangxi, Guizhou, Henan, Jiangsu, Anhui, and Fujian. Based on the local endemicity of falciparum malaria, each province/ autonomous region set pilot points in counties with high morbidity of falciparum malaria to enroll patients, and to make an in vivo four-week test [10] and an in vitro microtest [11,12]. Between 1981 and 1984, 466 cases from 23 counties were examined. Among them, 395 cases finished the treatment course and received observation four weeks later; 311 out of 321 cases had successful in vitro, and 224 cases were examined concurrently by in vivo and in vitro with success. The results indicated that chloroquine resistance had already existed in the eight provinces/autonomous regions, especially in Hainan and Yunnan where falciparum malaria was severely prevalent. In Hainan, 106 cases from four counties were examined by the in vivo test; 90 cases were successful, 74 were chloroquine resistant $(82.2 \%)$, and $35.1 \%$ had degree III resistance (RIII). Meanwhile, 123 cases from six counties were examined by the in vitro microtechnique; 120 cases were successful (86 cases were concurrently examined by the in vivo test), and 113 were chloroquine resistant (94.2\%), which was more severe in the southwestern mountainous area $[8,9,20]$. In Yunnan, 178 cases from four counties were examined by the in vivo four-week test; 155 were successful and 115 (74.2\%) had chloroquine resistance. Among 93 cases from four counties examined by the in vitro microtest, 88 cases were successful (81 cased were also examined by the in vivo test), 75 cases (85.2\%) were chloroquine resistant, and the resistance was severe in the bordering area of southern Yunnan [6,7]. In Guangxi, of the 46 cases examined by the in vivo test, 36 were successful and only 12 cases (33.3\%) were RI; 14 of the successful cases were sensitive. Among 28 cases examined by the in vitro microtest, 27 were successful and 21 (77.8\%) were chloroquine resistant. In Guizhou, 39 cases were examined by the in vivo test; 31 were successful, two (6.5\%) were RI. Among the 34 examined by the in vitro microtest, 33 were successful and 12 cases (36.4\%) appeared to show resistance. In Anhui, of the 25 cases examined by the in vivo four-week test, 20 were successful, four were RI, and 16 were RII. All 22 cases examined by the in vitro microtest developed resistance. In Henan, of the 62 cases examined by the in vivo four-week test, 52 were successful and three were RI. Of the nine cases examined by the in vitro test, three (33.3\%) were chloroquine resistant. In Jiangsu, eight cases examined by the in vivo four-week test were sensitive to chloroquine, but five out of 12 cases (41.7\%) examined by the in vitro microtest showed resistance. In Fujian, one of the two cases examined by the in vivo four-week test was RI. The results indicated that falciparum malaria highly resistant to chloroquine was present in Hainan and Yunnan provinces, and falciarum malaria in southern Guangxi and central Anhui also exhibited obvious chloroquine resistance, but the resistance level in these latter two provinces was lower than that in Hainan and Yunnan, and the chloroquine resistance in southern Henan, Guizhou, and western Jiangsu was at its initial stage [21] (see Table 1).

The above results indicated the corelationship between in vivo and in vitro tests. All 135 resistant cases determined by the in vivo test were also resistant by the in vitro test; and all 40 sensitive cases determined by the in vitro test also proved to be sensitive by the in vivo test. However, among the 95 sensitive cases determined by the in vivo test, 55 cases showed resistance at different levels by the in vitro test, indicating that some cases could not be detected by the in vivo test; thereby, the resistance rate by the in vitro test would often be higher than that by the in vivo test. At the same time, it could be seen that the higher the resistance rate by the in vivo test, the higher the drug concentrations for

Table 1 Result of the assessment of the chloroquine resistance of P. falciparum

\begin{tabular}{|c|c|c|c|c|c|c|}
\hline \multirow[t]{2}{*}{ Province } & \multicolumn{3}{|c|}{ In Vivo test } & \multicolumn{3}{|c|}{ In Vitro test } \\
\hline & No. cases examined & No. resistant cases & Resistance rate (\%) & No. cases examined & No. resistant cases & Resistance rate (\%) \\
\hline Hainan & 90 & 74 & 82.2 & 120 & 113 & 94.2 \\
\hline Yunnan & 155 & 115 & 74.2 & 88 & 75 & 85.2 \\
\hline Guangxi & 37 & 12 & 32.4 & 27 & 21 & 77.8 \\
\hline Guizhou & 31 & 2 & 6.5 & 33 & 12 & 36.4 \\
\hline Anhui & 20 & 20 & 100 & 22 & 22 & 100 \\
\hline Henan & 52 & 3 & 5.8 & 9 & 3 & 33.3 \\
\hline Jiangsu & 8 & 0 & 0 & 12 & 5 & 41.7 \\
\hline Fujian & 2 & 1 & 50.0 & & & \\
\hline
\end{tabular}


complete inhibition of schizont maturation by the in vitro test [16].

\section{Investigation on the fluctuation of the chloroquine resistance of $P$. falciparum}

Since chloroquine-resistant falciparum malaria was found in Yunnan and Hainan in 1973 and 1974, respectively, the resistant falciparum malaria began spreading rapidly, and the degree of resistance kept rising, becoming widely distributed from early individual resistant cases in just five years. In 1978, chloroquine-resistant P. falciparum was found in all 11 counties endemic for falciparum malaria in Hainan. Therefore, the Hainan provincial government issued a document to stop the use of chloroquine for malaria control from 1979 and replace it with piperaquine. In 1983, it was confirmed that chloroquine-resistant falciparum malaria spread all over the falciparum malaria endemic areas in Yunnan. Since then, chloroquine was rarely used, and the drugs mainly used for the control of falciparum malaria were artemisinins, pyronaridine, and the No. 3 antimalarial tablet (piperaquine + sulfadoxine). In order to understand the fluctuation of the chloroquine resistance of $P$. falciparum after stopping or reducing the use of chloroquine, the sensitivity of $P$. falciparum to chloroquine was investigated at one to three year intervals in the Hainan and Yunnan provinces.

\section{The results of the assessment in Hainan}

Variations in the chloroquine-resistance rate: A total of 287 cases from the Baoyou Town of Ledong County were examined eight times successively by the in vitro microtest; among them 128 cases were concurrently examined by the in vivo test. The resistance rate decreased from $97.7 \%$ in 1981 to $26.7 \%$ in 1997 . Taking the 1981 rate as 100 , this was a reduction of $72.7 \%(P<0.01)$. A total of 128 cases were examined by the in vivo fourweek test; the resistance rate decreased from $84.2 \%$ in 1981 to $18.4 \%$ in 1997 , a reduction of $78.1 \%(P<0.01)$. In 2001, 82 cases from the Yaliang Township of Sanya City were examined by the in vitro test; the resistance rate was 59.8\%. In 2003, 16 cases from the Fubao Township of Ledong County were examined by the in vivo four-week test; the resistance rate was $62.5 \%$, indicating that after stopping the use of chloroquine, the chloroquine resistance of P. falciparum followed a downward trend.

Variations in the chloroquine-resistance degree: The results by the in vitro microtest demonstrated that the mean drug concentration for complete inhibition of schizont maturation decreased from $10.4 \pm 7.1 \mathrm{pmol} / \mu \mathrm{l}$ blood in 1981 to $1.6 \pm 1.5 \mathrm{pmol} / \mu \mathrm{l}$ blood in 1997 , a reduction of $84.4 \%(P<0.01)$. This indicated that in most cases, as the time of stopping the use of chloroquine extended, the necessary drug concentration for complete inhibition of schizont maturation decreased gradually from the previous higher drug concentration $(>6.4 \mathrm{pmol} / \mu \mathrm{l})$ to a lower drug concentration $(<1.6 \mathrm{pmol} / \mu \mathrm{l})$. During 1981-1997, the former decreased from $83.3 \%$ to $6.7 \%$, a reduction of $92.0 \%(P<0.01)$, and the latter increased from $4.2 \%$ to $73.3 \%$, an increase of $94.3 \%(P<0.01)$. The result of the assessment by the in vivo four-week test demonstrated that the asexual parasite clearance time in the blood reduced from 72 hours in 1981 to 50.7 hours in 1997, the percentage of RIII cases in the whole spectrum of resistant cases reduced from 53.1\% in 1981 to $14.3 \%(P<0.01)$ in 1997 . In 2001,82 cases from the Yaliang Township of Sanya City were examined by the in vitro microtest; the results indicated that the mean drug concentration for complete inhibition of schizont maturation was $3.56 \mathrm{pmol} / \mu \mathrm{l}$ blood and in $12.5 \%$ cases, it was $>6.4 \mathrm{pmol} / \mu \mathrm{l}$ blood. In 2003, 16 cases from the Fubao Township of Ledong County were examined by the in vivo four-week test; the results indicated that the asexual parasite clearance time in the blood was an average of 56.9 hours and the percentage of RIII cases was $20 \%$, indicating that the degree of the chloroquine resistance of $P$. falciparum reduced gradually after the use of chloroquine was stopped [22-26].

\section{The results of the assessment in Yunnan}

Variations in the chloroquine-resistance rate: A total of 234 cases from the Mengla County were examined seven times successively by the in vitro microtest; the resistance rate decreased from $97.4 \%$ in 1981 to $77.8 \%$ in 1999 , a reduction of $20.1 \%(P<0.01)$. A total of 27 cases from the Jinghong County were examined by the in vitro microtest; the resistance rate was $70.4 \%$.

Variations in the chloroquine-resistance degree: The mean drug concentration for complete inhibition of schizont maturation decreased from $17.2 \pm 12.6 \mathrm{pmol} / \mu \mathrm{l}$ blood in 1981 to $4.4 \pm 3.1 \mathrm{pmol} / \mu \mathrm{l}$ blood in 1999 , a reduction of $74.4 \%(P<0.01)$. The percentage of the cases with a complete inhibition of schizont maturation in the drug concentration $>6.4 \mathrm{pmol} / \mu \mathrm{l}$ in blood decreased from $58.9 \%$ in 1981 to $19.6 \%$ in 1999 , a reduction of $66.7 \%(P<0.01)$. In 2002, 27 cases from the Jinghong County were examined; the mean drug concentration for complete inhibition of schizont maturation was $4.0 \pm 3.3 \mathrm{pmol} / \mu \mathrm{l}$ blood, and $16.6 \%$ cases had complete inhibition of schizont maturation in drug concentration $>6.4 \mathrm{pmol} / \mu \mathrm{l}$ in blood, indicating that the chloroquine-resistance rate and degree of $P$. falciparum fell gradually after reduced use of chloroquine [26] (see Table 2).

\section{Present situation of the sensitivity of $P$. falciparum to antimalarial drugs}

Due to the widespread chloroquine resistance of P. falciparum, application of other antimalarial drugs increased from 1980. In order to understand the sensitivity of the 
Table 2 Variations in the chloroquine resistance of $P$. falciparum (In vitro)

\begin{tabular}{lccccc}
\hline Province & Year & $\begin{array}{c}\text { No. of } \\
\text { cases } \\
\text { examined }\end{array}$ & $\begin{array}{c}\text { Resistance } \\
\text { rate (\%) }\end{array}$ & $\begin{array}{c}\text { Mean inhibition } \\
\text { concentration } \\
(\boldsymbol{p m o l} / \boldsymbol{\mu} /)^{*}\end{array}$ & $\begin{array}{c}>\text { 6.4 pmol/ } \\
\boldsymbol{\mu} \text { *** }\end{array}$ \\
\hline Hainan & 1981 & 48 & 97.7 & $10.46 \pm 7.14$ & 83.3 \\
& 1997 & 45 & 26.7 & $1.63 \pm 1.17$ & 6.7 \\
Yunnan & 1981 & 39 & 97.4 & $17.2 \pm 12.6$ & 58.9 \\
& 1999 & 18 & 77.8 & $4.4 \pm 3.1$ & 19.5 \\
\hline
\end{tabular}

*The mean drug concentration for complete inhibition of schizont maturation. **Percentage of cases with complete inhibition of schizont maturation.

parasite to commonly used antimalarials and to guide rational administration of the drugs, the sensitivity of P. falciparum to chloroquine, amodiaquine, piperaquine, mefloquine, pyronaridine, artesunate, arteether, dihydroartemisinin, and quinine was examined three and five times in Hainan and Yunnan, respectively, between 1984 and 2002. The results indicated that the $P$. falciparum in the two provinces had developed resistance to seven antimalarial drugs except for mefloquine and quinine, and displayed relatively higher resistance to chloroquine, amodiaquine, and piperaquine. However, the sensitivity of $P$. falciparum to chloroquine was recovering. The rate and degree of resistance to piperaquine were on the rise. In the Hainan Province, a total of 216 cases were examined by the in vitro test five times successively; the resistance rate increased from $15.8 \%$ in 1985 to $72.9 \%$ in 1997, and the mean drug concentration for complete inhibition of schizont maturation increased from $9.7 \mathrm{pmol} / \mu$ in 1985 to $47.9 \mathrm{pmol} / \mu \mathrm{l}$ in 1997 , which was almost five times higher than it was in 1985. A total of 154 cases were examined by the in vivo test three times succesively; the resistance rate increased from $17.2 \%$ in 1984 to $50.0 \%$ in 1997. No RIII cases were found in 1984, but RIII cases accounted for $71.4 \%$ of the total resistant cases in 1997. In the Yunnan Province, 126 cases were examined by the in vitro test three times successively; the resistance rate increased from $21.3 \%$ in 1990 to $73.0 \%$ in 1993 , and the mean drug concentration for complete inhibition of schizont maturation increased from $19.0 \mathrm{pmol} / \mu \mathrm{l}$ in 1990 to $38.0 \mathrm{pmol} / \mu \mathrm{l}$ in 1993 , which was two to three times higher than in 1990. The results of clinical treatment indicated that $50 \%$ of falciparum malaria cases in Hainan developed resistance to piperaquine (see Table 3), and the sensitivity to pyronaridine and artemisinin derivatives was falling gradually. Even artemether-resistant cases were found through clinical treatment in Yunnan [27-32]. In Hainan, the mean concentration of pyronaridine and artesunate for complete inhibition of schizont maturation in vitro increased by four to eight times and two to eight times, respectively [33-37] (see Table 4).

\section{Rational use of antimalarial drugs in China}

After completion of the survey on the resistance degree of chloroquine-resistant falciparum malaria and its geographical distribution, the results and suggestions for improving local malaria control were reported to the Ministry of Health and the local governments, recommening that surveillance on drug-resistant malaria be listed as one of the important tasks in malaria control. For those provinces/autonomous regions only with sporadic falciparum malaria cases, it was suggested that mosquito control measures were strengthened to prevent possible focal transmission of falciparum malaria, focusing more on migrants to prevent the spreading of the drug resistance more effectively. In Hainan and Yunnan, where critical drug resistance persisted, use of chloroquine for falciparum malaria control should be stopped, and the therapeutic effect of other substitutes should be monitored, in order to find and contain the new development of drug resistance in a timely fashion. Due to active implementation of the control program, the number of falciparum malaria endemic provinces/autonomous regions decreased yearly, and after 1998, falciparum malaria was prevalent only in the Hainan and Yunnan provinces.

In order to more effectively cure malaria patients and avoid or delay the development of drug resistance of $P$. falciparum, principles and therapeutic regimens for the application of antimalarial drugs in China were formulated in 2000 on the basis of the drug sensitivity of the parasite found through the surveys. They were divided into first-line, second-line, and third-line drugs, in order to make a reasonable and standardized application of the drugs. Chloroquine and piperaquine were recommended as the first-line drugs for endemic areas of vivax

Table 3 Sensitivity of $P$. falciparum to piperaquine

\begin{tabular}{|c|c|c|c|c|c|c|c|c|}
\hline \multirow[t]{2}{*}{ Province } & \multicolumn{4}{|c|}{ In vitro test } & \multicolumn{4}{|c|}{ In vivo test } \\
\hline & Year & $\begin{array}{l}\text { No. of cases } \\
\text { examined }\end{array}$ & Resistance rate (\%) & $\begin{array}{l}\text { Inhibition concentration } \\
(\mathrm{pmol} / \mu /)^{*}\end{array}$ & Year & No. of cases examined & Resistance rate (\%) & RIII $(\%)^{* *}$ \\
\hline \multirow[t]{2}{*}{ Hainan } & 1985 & 38 & 15.5 & $9.7 \pm 9.1$ & 1984 & 64 & 17.2 & 0 \\
\hline & 1997 & 70 & 72.9 & $47.9 \pm 36.5$ & 1997 & 42 & 50.0 & 71.4 \\
\hline \multirow[t]{2}{*}{ Yunnan } & 1990 & 47 & 21.3 & $19.0 \pm 17.2$ & & & & \\
\hline & 1993 & 37 & 73.0 & $38.0 \pm 32.5$ & & & & \\
\hline
\end{tabular}

*The mean concentration of piperaquine for complete inhibition of schizont maturation.

**Percentage of RIII cases in total resistant cases. 
Table 4 Sensitivity of P. falciparum to pyronaridine and artesunate

\begin{tabular}{|c|c|c|c|c|c|c|c|c|}
\hline \multirow[t]{2}{*}{ Drug } & \multirow[t]{2}{*}{ Province } & \multicolumn{3}{|c|}{ In vitro test } & \multicolumn{4}{|c|}{ In vivo test } \\
\hline & & Year & $\begin{array}{l}\text { No. of cases } \\
\text { examined }\end{array}$ & $\begin{array}{l}\text { Inhibition concentration } \\
(\mathrm{pmol} / \mu /)^{*}\end{array}$ & Year & $\begin{array}{l}\text { No. of cases } \\
\text { examined }\end{array}$ & $\begin{array}{l}\text { Defervescence } \\
\text { time }(h)\end{array}$ & $\begin{array}{c}\text { Parasite clearance } \\
\text { time }(h)\end{array}$ \\
\hline \multirow[t]{3}{*}{ Pyronaridine } & Hainan & 1986 & 20 & 0.08 & 1986 & 42 & $32.9 \pm 12.4$ & $43.3 \pm 23.3$ \\
\hline & & 1997 & 37 & 1.36 & 1996 & 13 & $40.2 \pm 15.7$ & $62.5 \pm 28.7$ \\
\hline & Yunnan & 1988 & 25 & 0.14 & & & & \\
\hline \multirow[t]{4}{*}{ Artesunate } & Hainan & 1986 & 18 & 0.028 & & & & \\
\hline & & 2002 & 32 & 0.135 & & & & \\
\hline & Yunnan & 1988 & 24 & 0.038 & & & & \\
\hline & & 2002 & 22 & 0.054 & & & & \\
\hline
\end{tabular}

*The mean drug concentration for complete inhibition of schizont maturation.

malaria and some areas of falciparum malaria where chloroquine and piperaquine were still sensitive. Artemisinin derivatives and pyronaridine were recommended as the second-line drugs for areas of falciparum malaria with moderate or high resistance to chloroquine and piperaquine. Artemisinin derivatives or pyronaridine in combination with other antimalarials were recommended as the third-line drugs for those areas where the therapeutic effect of second-line drugs was limited [38-46]. Considering that $P$. falciparum in China has generally developed resistance to chloroquine, piperaquine and sulfadoxinepyrimethamine, and other antimalarials, artemisinin derivatives and pyronaridine have been widely used as the first-line drugs in endemic areas of falciparum malaria. Due to the rational and standardized use of antimalarials, the therapeutic effect was greatly improved, malaria cases decreased considerably from 903,802 cases in 1984 to 7,855 cases in 2010 (including 1,258 falciparum malaria cases), and the number of falciparum malaria endemic counties decreased from 74 in eight provinces in 1984 to just 17 in the Yunnan Province only in $2010[47,48]$.

\section{Conclusion}

Drug resistance of $P$. falciparum is a global problem, especialy in Southeast Asia. In some neighboring countries, such as Thailand, Cambodia, Myanmar, India, and Vietnam, P. falciparum has developed resistance to chloroquine, sulfadoxine-pyrimethamine, mefloquine, etc., extensively. On the Thailand-Cambodia border and in Myanmar, high multiple resistance has appeared [40,49], and Myanmar and Thailand have reported that $P$. vivax has developed resistance to chloroquine and primaquine, respectively $[4,5]$. Therefore, antimalarials have been devided into first-line drugs and second-line drugs for rational application in these countries [40]. Chloroquine, piperaquine, pyronaridine, and artemisinin derivatives are commonly used antimalarials in China. Chloroquine has been used extensively for more than 50 years, and the others are relatively new antimalarials and have been used for over 20 years.
The results of the survey indicated that there was a certain variability of the resistance of $P$. falciparum to chloroquine-the resistance rate and degree to chloroquine was increasing from the appearance of chloroquineresistant falciparum malaria cases to the early stage of reducing or stopping the use of chloroquine. Investigations indicated that once $P$. falciparum developed resistance to chloroquine, when mosquito vectors with strong transmissibility existed (such as Anopheles dirus and An. minimus), and with frequent human population movement, falciparum malaria would spread rapidly. Once resistance to chloroquine appeared, if the use of chloroquine could be stopped in a timely manner, the parasite could gradually restore its sensitivity to chloroquine under the condition of no drug pressure, but the recovery progress would be slow. In the Hainan Province, 18 years after stopping the use of chloroquine, about $20-60 \%$ of the cases still had certain resistance to chloroquine. The process of recovery of chloroquine sensitivity firstly indicated a decline of the resistance degree, then the reduction of the resistance rate when the resistance level dropped below a certain level. In the first 10 years after stopping use of chloroquine, the resistance degree fell faster, yet the resistance rate hardly changed; however, 10 more years later, the resistance rate fell faster as well.

In the period of extensive use of piperaquine, pyronaridine, artemisinin derivatives, or the No. 3 antimalarial tablet, there were plenty of cases taking insufficient dosages due to low compliance, which was also one of the major reasons for a decrease in the sensitivity to those drugs. It was not recommended to use artemisinin derivatives and pyronaridine in endemic areas of vivax malaria; neither for treatment, preventive medicine, or presumptive treatment for individual cases. They were always sensitive and effective clinically, yet the sensitivity of P. falciparum to the drugs was in a gradual downward trend. In order to delay the development of resistance and protect antimalarial drugs, based on the WHO advocation to use combination artemisinin drugs, it was suggested 
not to use artemisinin derivatives alone, but in combination with other antimalarials $[40,50,51]$.

\section{Additional file}

\section{Additional file 1: Multilingual abstracts in the six official working} languages of the United Nations.

\section{Competing interests}

The author declares that they have no competing interests.

\section{Acknowledgements}

The author gratefully acknowledges Mr. Chen Pulin for helping with the English translation of the manuscript.

Received: 27 November 2013 Accepted: 11 February 2014 Published: 24 February 2014

\section{References}

1. Payne D: Spread of chloroquine resistance in Plasmodium falciparum J. Parasitol Today 1987, 3:241-246.

2. Suebsaeng $L$, Wernsdorfer WH, Rooney W: Sensitivity to quinine and mefloquine of Plasmodium falciparum in Thailand. Bull WHO 1986 64:759-765.

3. ter Kuile FO, Dolan G, Nosten F, Edstein MD, Luxemburger C, Phaipun L, Chongsuphajaisiddhi T, Webster HK, White NJ: Halofantrine versus mefloquine in treatment of multidrug-resistant falciparum malaria. Lancet 1993, 341:1044-1049.

4. Murphy GS, Basri H, Purnomo, Andersen EM, Bangs MJ, Mount DL, Gorden J, Lal AA, Purwokusumo AR, Harjosuwarno S, Hoffman SL: Vivax malaria resistant to treatment and prophylaxis with chloroquine. Lancet 1993, 341:96-100.

5. Looareesuwan S, Buchachart K, Wilairatana P, Chalermrut K, Rattanapong Y, Amradee S, Siripiphat S, Chullawichit S, Thimasan K, Ittiverakul M, Triampon A, Walsh DS: Primaquine-tolerant vivax malaria in Thailand. Ann Trop Med Parasitol 1997, 91:939-943.

6. Che LG, Chen WC, Yang HL, Huang KG: Report on the survey of geographical distribution of chloroquine-resistant strain of Plasmodium falciparum in Yunnan province. Chin J Epidemiol 1986, 7(2):88-91. in Chinese.

7. Yang $H L$, Yang PF, Dong Y, Che LG, Liu DQ, Liu RJ, Zhan B, Zhang CY, Gao DQ: Longitudinal surveillance of the resistance of Plasmodium falciparum to chloroquine in Yunnan Province. Chin J Parasitol Parasitic Dis 1994, 12 (1):31-33. in Chinese.

8. Cai $X Z$, Pang $X J$, Chen WJ, Li H, Ren DX: Investigation on the sensitivity of Plasmodium falciparum to chloroquine at Qianjia, Hainan Island. Chin J Prev Med 1982, 16(1):6-9. in Chinese.

9. Cai XZ, Pang XJ, Zeng QQ, Chen WJ, Wang XF, Zhang HJ, Wu Z, Huang QL, Ren DX, Liu DQ, Qiu CP: The result of 10-year investigation of chloroquine-resistant Plasmodium falciparum in Hainan Island. Chin $\rfloor$ Epidemiol 1986, 7(2):92-95. in Chinese.

10. WHO Technical Report Series: Chemotherapy of malaria and resistance of antimalarials. Geneva: Report of a WHO Scientific Group; 1973.

11. Rieckmann $\mathrm{KH}$, Campbell GH, Sax LJ, Mrema JE: Drug sensitivity of Plasmodium falciparum. An in-vitro microtechnique. Lancet 1978, 1:22-23.

12. WHO: Instruction for use of the microtest kit for the assessment of the response of Plasmodium falciparum to chloroquine and mefloquine in vitro. Geneva; 1981.

13. Division of Control of Tropical Diseases: World Health Organisation. In In vitro micro-test (Mark III) for assessment of the response of Plasmodium falciparum to chloroquine, mefloquine, quinine, amodiaquine, sulfadoxine/ pyrimethamine and artemisinin. World Health Organization CTD/MAL/97.20 Rev; 2001:2.

14. World Health Organization: Assessment and monitoring of antimalarial drug efficacy for the treatment of uncomplicated flaciparum malaria. WHO/HTM/ $\mathrm{RBM} / ; 2003: 50$

15. World Health Organization: Recommended Genotyping Procedures (RGPs) to identify parasite populations. In Developed after an informal consultation organized by the Medicines for Malaria Venture and cosponsored by the World Health Organization. Amsterdam, Netherland; 2007

16. Liu DQ, Ren DX, Liu RJ: Preparation of the lyophilized culture medium for in vitro microtest of chloroquine-resistant Plasmodium falciparum and improvement of chloroquine-coated plate. J Parasitol Parasitic Dis 1983, 1 (1):44-48. in Chinese.

17. Liu DQ, Ren DX, Liu RJ, Qiu CP, Gao DQ: A culture medium easy for field test on the drug resistance of Plasmodium falciparum. Chin J Parasitol Parasitic Dis 1989, 7(2):112-113. in Chinese.

18. Liu DQ, Ren DX Qiu CP, Liu RJ: Comparison of the efficacy of two culture media for in vitro microtest on the sensitivity of Plasmodiumfalciparum to chloroquine and mefloquine. Chin J Parasitol Parasitic Dis 1986, 4 (4):256-258. in Chinese.

19. Ren DX, LiU DQ, Liu RJ: Sensitivity of Plasmodium falciparum in Hainan Island to chloroquine by the in vitro microtechnique. Acta Microbio/ Sin 1981, 21:510-512. in Chinese.

20. Huang QL, Zhu Z, Ren DX, Liu DQ, Qiu CP, Liu RJ, Lin CF, Yang XP: A successive survey on the sensitivity of Plasmodium falciparum patients to chloroquine by in vitro microtechnique and in vivo test in Hainan Island. Chin J Prev Med 1985, 19(4):220-222. in Chinese.

21. Liu DQ, Qiu CP, Liu RJ, Ren DX: Resistance degree of Plasmodium falciparum to chloroquine and its geographical distribution in China. Chin J Parasitol Parasitic Dis 1986, 4(2):81-85. in Chinese.

22. Onori $E, V u$ Thi $P$ : Is Plasmodium falciparum resistance to chloroquine reversible in absence of drug pressure? Lancet 1986, 1:319.

23. Liu DQ, Liu RJ, Ren DX, Gao DQ, Zhang CY, Qiu CP, Cai XZ, Lin CF, Liang AH: Alteration in resistance of Plasmodium falciparum to chloroquine after cessation of chloroquine medication for twelve years. Chin J Parasitol Parasitic Dis 1992, 12(4):241-244. in Chinese.

24. Liu DQ, Liu RJ, Ren DX, Gao DQ, Zhang CY, Qui CP, Cai XZ, Ling CF, Song $\mathrm{AH}$, Tang $\mathrm{X}$ : Changes in the resistance of Plasmodium falciparum to chloroquine in China. Bull World Health Organ 1995, 73:483-486.

25. Liu DQ, Cai XZ, Ren DX, Liu RJ, Lin SG, Zeng LH, Tang X: Changes in chloroquine resistance of Plasmodium falciparum in Hainan province. Chin J Parasitol Parasitic Dis 1999, 17(1):32-34. in Chinese.

26. Liu DQ, Feng XP, Yang HL, Lin SG, Chen WJ, Yang PF: Fluctuation in the resistance of Plasmodium falciparum to chloroquine in China. Chin J Parasitol Parasitic Dis 2005, 23(1):27-31. in Chinese.

27. Yang $\mathrm{HL}, \mathrm{He} \mathrm{H}$ : A report of one artemether-resistant falciparum malaria case. Chin J Parasitol Parasitic Dis 1991, 9(2):125. in Chinese.

28. Yang HL, Yang PF, Liu DQ, Liu RJ, Dong Y, Zhang CY, Gao DQ, He H: In vitro test on the sensitivity of Plasmodium falciparum to chloroquine, pyronaridine, srtesunate and piperaquine in south Yunnan. Chin $J$ Parasitol Parasitic Dis 1992, 10(3):198-200. in Chinese.

29. Yang HL, Yang PF, Yang YM: In vitro test on the sensitivity of Plasmodium falciparum to mefloquine, quinine, amodiaquine, chloroquine, sulfadoxine/pyrimethamine and pyronaridine in south Yunnan. Chin $J$ Parasitol Parasitic Dis 1994, 12(2):140-142. in Chinese.

30. Yang $\mathrm{HL}$, Liu DQ, Huang $\mathrm{KG}$, Yang $\mathrm{YM}$, Yang PF, Liao MZ, Zhang $\mathrm{CY}$ : In vitro assay of the sensitivity of Plasmodium falciarum to chloroquine, amodiaquine, piperaquine, mefloquine and quinine in Yunnan province. Chin J Parasitol Parasitic Dis 1999, 17(1):43-45. in Chinese.

31. Yang HL, Liu DQ, Huang KG, Yang PF, Yang YM, Liao MZ, Zhang CY, Liu RJ: In vitro assay of the sensitivity of Plasmodium falciparum to the derivatives of artemisimin, pyronaridine and chloroquine in Yunnan. Chin J Parasitol Parasitic Dis 1997, 15(5):292-296. in Chinese

32. Yang HL, Liu DQ, Huang KG, Zhang CY, Li CF: Longitudinal surveillance of the sensitivity of Plasmodium falciparum to pyronaridine in south Yunnan. Chin J Parasitol Parasitic Dis 1998, 16(2):81-83. in Chinese

33. Cai XZ: Investigation of piperaquine resistance of Plasmodium falciparum and its treatment in Hainan Island. Hainan Health 1989, 17(4):4-8. in Chinese.

34. Wu Z, Huang QL, Zhang KY: Piperaquine resistance of Plasmodium falciparum in Hainan Island. Chin Med J (Engl) 1985, 65:483.

35. Zhang KY, Zhou JX, Wu Z, Huang QL: In vitro assay on the sensitivity of Plasmodium falciparum to chloroquine, piperaquine, amodiaquine, mefloquine and quinine. Chin J Parasitol Parasitic Dis 1987, 5(3):165. in Chinese.

36. Qiu CP, Liu DQ, Liu RJ, Ren DX: Surveillane on sensitivity of Plasmodium falciparum to pyronaridine and artesunate. Chin J PPrev Med 1982, 22:368. in Chinese. 
37. Liu DQ, Liu RJ, Zhang CY, Cai XZ, Tang X, Yang HL, Yang PF, Dong Y: Present situation on the sensitivity of plasmodium falciparum to antimalarial drugs in China. Chin J Parasitol Parasitic Dis 1996, 14(1):37-41. in Chinese.

38. WHO Regional Office for the Western Pacific: Resistance of Plasmodium falciparum and policies for treatment of falciparum malaria. In Proceeding of the Informal consultation on monitoring resistance to antimalarial drugs in the Mekong region. Phnom Penh, Cambodia; 2000:16-17.

39. WHO: The use of artermisinin and its derivatives as antimalarial drugs (Archived). Geveva: Report of a Joint CTD/DMP/TDR Informal Consultation; 1998:10-12.

40. Roll Back Malaria/WHO: The use of antimalarial drugs. Geveva: Report of a WHO Info Consultation; 2000:13-17.

41. Roll Back Malaria/WHO: Antimalarial drug combination therapy. Geveva: Report of a WHO Tech Consultation; 2001:4-5.

42. Bunnag D, Karbwang J, Harinasuta T: Artemether in the treatment of multiple drug resistant falciparum malaria [J]. Southeast Asian J Trop Med Public Health 1992, 23:762-767.

43. Liu DQ, Lin SG, Feng XP, Chen WJ, Chen FL, Wu HM, Chen C, Liu J: Study on treatment of multi-drug resistant falciparum malaria by using a combination of dihydroartemisinin and pyronaridine. Chin J Parasitol Parasitic Dis 2002, 20(4):193-196. in Chinese.

44. Huang QL, Ouyang WC, Zhou JX, Wu Z, Zhang KY, Huang JK, Cai XZ, Pang XJ, Fu SG, Wang XF, Liu J: Effectiveness of amodiaquine, sulfadoxinepyrimethamine and combinations of these drugs for treating chloroquine-resistant falciparum malaria in Hainan Island, China. Bull World Health Organ 1988, 66(3):353-358.

45. Che LG, Zhang YL, Li XL, Shu WY, Zhang X, Li CF, Liu ZH: Clinical study on the treatment of drug-resistant Plasmodium falciparum with compound artemether tablet. Chin J Parasitic Dis Control 1995, 8(4):244-245. in Chinese.

46. Expert Advisory Committee on Malaria, Ministry of Health, China: Principles for antimalarial drug use in China. Chin J Parasitic Dis Control 2002, 15 (3):129-130. in Chinese.

47. Malaria Commission, Committee of Medical Sciences: Malaria situation in the People's Republic of China in 1984. Chin J Parasitol Parasitic Dis 1985, 3(4):241-243. in Chinese.

48. Zhou SS, Wang Y, Li Y: Malaria situation in the People's Republic of China in 2010. Chin J Parasitol Parasitic Dis 2011, 29(6):401-403. in Chinese.

49. Bloland PB: Drug resistance in malaria. WHO/CDS/CSR/DRS 2001, 10:6-8.

50. White NJ: Preventing antimalarial drug resistance through combinations. Drug Res Updates 1998, 1:3-9.

51. White NJ: Antimalarial drug resistance and combination chemotherapy. Philos Trans R Soc Lond B Biol Sci 1999, 354:739-749.

doi:10.1186/2049-9957-3-8

Cite this article as: Liu: Surveillance of antimalarial drug resistance in China in the 1980s-1990s. Infectious Diseases of Poverty 2014 3:8.

\section{Submit your next manuscript to BioMed Central and take full advantage of:}

- Convenient online submission

- Thorough peer review

- No space constraints or color figure charges

- Immediate publication on acceptance

- Inclusion in PubMed, CAS, Scopus and Google Scholar

- Research which is freely available for redistribution

Submit your manuscript at www.biomedcentral.com/submit
() Biomed Central 